\title{
Identification of muscle weakness in older adults from normalized upper and lower limbs strength: a cross-sectional study
}

\author{
Pedro Pugliesi Abdalla 1,2*, Lucimere Bohn ${ }^{2,6}$, Leonardo Santos Lopes da Silva ${ }^{3}$, André Pereira dos Santos ${ }^{1}$, \\ Marcio Fernando Tasinafo Junior ${ }^{3}$, Ana Claudia Rossini Venturini ${ }^{1}$, Anderson dos Santos Carvalho ${ }^{4}$, \\ David Martinez Gomez ${ }^{5}$, Jorge Mota ${ }^{2}$ and Dalmo Roberto Lopes Machado 1,2,3
}

\begin{abstract}
Background: To propose cut-off points for older adults' weakness for upper and lower limbs muscle strength normalized by body size with the ratio standard/muscle quality and allometric scaling.

Methods: Ninety-four community-dwelling older adults (69.1\% women) were assessed for 49 body-size variables (anthropometry, body composition and body indexes), handgrip strength (HGS), one maximum repetition measurement for knee extensors (1RM), isokinetic knee extension peak torque at $60^{\circ} / \mathrm{s}(\mathrm{PT})$, and six-minute walk test (6MWT). Ratio standard or muscle quality (muscle strength/body size) and allometric scaling (muscle strength/body size ; when ${ }^{b}$ is the allometric exponent) were applied for body-size variables significantly correlated with HGS, 1 RM and PT. Cut-off points were computed according to sex based on mobility limitation ( $6 \mathrm{MWT}<400 \mathrm{~m}$ ) with ROC curve and Youden index.
\end{abstract}

Results: Absolute HGS, 1RM and PT cut-off points were not adequate because they were associated with body size $(r>0.30)$. But it was corrected with muscle strength normalization according to body size-variables: HGS $(n=1)$; $1 R M(n=24)$ and PT $(n=24)$. The best cut-off points, with the highest area under the curve (AUC), were found after normalization for men: HGS/forearm circumference $(1.33 \mathrm{~kg} / \mathrm{cm}, A \cup C=0.74), 1 \mathrm{RM} /$ triceps skinfold $(4.22 \mathrm{~kg} / \mathrm{mm}$, $A U C=0.81)$, and PT/body mass*height ${ }^{0.43}\left(13.0 \mathrm{Nm} / \mathrm{kg}^{*} \mathrm{~m}^{0.43}, \mathrm{AUC}=0.94\right)$; and for women: HGS/forearm circumference $(1.04 \mathrm{~kg} / \mathrm{cm}, A \cup C=0.70), 1 \mathrm{RM} /$ body mass $(0.54 \mathrm{~kg} / \mathrm{kg}, A \cup C=0.76)$; and PT/body mass ${ }^{0.72}\left(3.14 \mathrm{Nm} / \mathrm{kg}^{0.72}\right.$; $\mathrm{AUC}=0.82$ ).

Conclusions: Upper and lower limbs muscle weakness cut-off points standardized according to body size were proposed for older adults of both sexes. Normalization removes the effect of extreme body size on muscle strength (both sexes) and improves the accuracy to identify weakness at population level (for women, but not in men), reducing the risk of false-negative/positive cases.

Keywords: Allometrically scaled, Disability, Evaluation, Frailty, Function/functional status, Measurement, Sarcopenia, Scaling

*Correspondence: pedroabdalla11@gmail.com

${ }^{1}$ College of Nursing at Ribeirão Preto, University of São Paulo, Ribeirão Preto, SP, Brazil

Full list of author information is available at the end of the article

\section{Background}

Muscle weakness is a natural muscle strength loss occurring along aging, and it predicts older adults' increased risk of hospital admissions, depression, fractures and premature mortality [1-3]. Muscle weakness 
can predict functional disability (i.e., difficulty to perform instrumental and basic activities of daily livingADL) like as mobility limitation [4], which is even more important than multimorbidity to forecast mortality amongst older adults [5]. As a consequence of its predictive ability, muscle weakness was used to identify geriatric syndromes such as dynapenia [6], frailty [7] and sarcopenia [8].

Muscle weakness is normally measured using muscle strength tests such as handgrip (HGS) or leg extension strength [8]. The current values to identify muscle weakness are based on absolute (non-normalized) muscle strength results $[7,9-17]$ or dividing absolute results by a body-size variable (ratio standard) such as body mass $[18,19]$ or by some body composition component, like lean tissue (muscle quality) [20-22]. The identification of weakness based on absolute muscle strength cutoff points may be inaccurate for lighter body mass and shorter height older adults [23-25]. In fact, the absolute values characterize lighter and shorter body size older adults as having muscle weakness, even if they sustain their instrumental and basic ADL [26]. This is a false positive muscle weakness diagnostic, that frequently leads to an unnecessarily utilization of public health resources, contributing to health burden [27]. Another topic that merits consideration is the inaccuracy of the ratio standard/muscle quality procedure because it overestimates the real strength of light/short older adults and underestimates it for tall/heavy ones [26]. These limitations are a consequence of the nonlinear relationship between muscle strength and body-size variables [23-25]. To overcome these constraints, the utilization of allometric scaling, that contemplates power and sensitivity in the nonlinear relationship between muscle strength and body size with the allometric exponent $\left({ }^{b}\right)$ might represent an adequate option [23-26].

Previous studies reported already the power function ratio in older adults between HGS and body-size variables as body mass ( ${ }^{b=0.63}$ or ${ }^{0.40}$ or ${ }^{0.31}$ ) [23-25], height $\left({ }^{b=1.84}\right)$ [24] and fat-free mass (FFM) $\left({ }^{b=0.46}\right)$ [24] and between leg extension strength and body mass $\left({ }^{\mathrm{b}=0.67}\right.$ or 0.69 or ${ }^{0.72}$ or ${ }^{0.74}$ or $\left.{ }^{0.96}\right)[26,28]$. Indeed, scaling HGS by body size (example: HGS/heigth ${ }^{1.84}$ ) removes the effect of body size on muscle strength [24], but the scaling muscle strength by body size to determine muscle weakness cut-off points has not been considered from HGS and knee extension in isokinetic dynamometer, excepting the one maximum repetition measurement for knee extensors (1RM) scaled to body mass [26]. Besides, important body-size variables related to mobility and ADL (e.g. fat mas [29], FFM [30] and leg length [31]) were not utilized to scaling muscle strength and create muscle weakness cut-off points.
Thus, our objective is to propose cut-off points for older adults' weakness with upper and lower limbs muscle strength normalized by body-size with the ratio standard/muscle quality and allometric scaling. We hypothesize that the normalization of muscle strength by ratio standard/muscle quality and allometry can be a way to approach muscle strength regardless of body size, which should reduce the risk of bias in identifying falsepositive cases of vulnerable older people.

\section{Method}

\section{Design and study population}

This is a cross-sectional study conducted from October 2016 to May 2017 at the University Hospital of Ribeirao Preto School of Medicine, University of São Paulo, Brazil (HC-FMRP-USP). The study was approved by the HC-FMRP-USP institutional review board (CAAE: 54345016.6.3001.5440). Older adults were voluntarily recruited and assigned an informed consent. This manuscript followed the guidelines from The Strengthening the Reporting of Observational Studies in Epidemiology (STROBE) conference list [32].

The sample consisted of 94 community-dwelling older adults ( $\geq 60$ years old, $69.1 \%$ women) recruited in projects for older adults of USP and in health community services. Inclusion criteria were $\geq 60$ years old, walk independently, absent limitation to execute all procedures, acute infections, cancer diagnosis, hip or knee prostheses, unstable cardiovascular condition, stroke sequelae, tumors, and weight loss $>3 \mathrm{~kg}$ in the last three months. The exclusion criteria were discontinuity in the study and cognition impairment (assessed by Mini Mental State Examination).

A sample size calculation $\left(\mathrm{n}=[\mathrm{ZySD} / \varepsilon]^{2}\right)$ [33] with trust level $(\mathrm{Zy}=0.95)$, greater compatible population variability founded in the literature (SD of $1 \mathrm{RM}: \pm 19.96 \mathrm{~kg}$ ) $[34,35]$ and maximum desired error $(\varepsilon \leq 8.0 \mathrm{~kg})$ was performed and identified a minimum sample size of $n=24$ for each sex.

\section{Procedures}

A multidisciplinary health team (nurses, nutritionists, pharmacists, physical educators, physicians, and physiotherapists) performed data collection. The appraisers were the same in each test. Data collection occurred on three non-consecutive days: 1st) recruitment: inclusion criteria verification by phone calls; 2 nd) cognition assessment, anthropometrics, body composition, HGS, mobility and physical activity level assessment; and 3rd) lower limbs muscle strength assessment. These procedures are resumed in Fig. 1. 


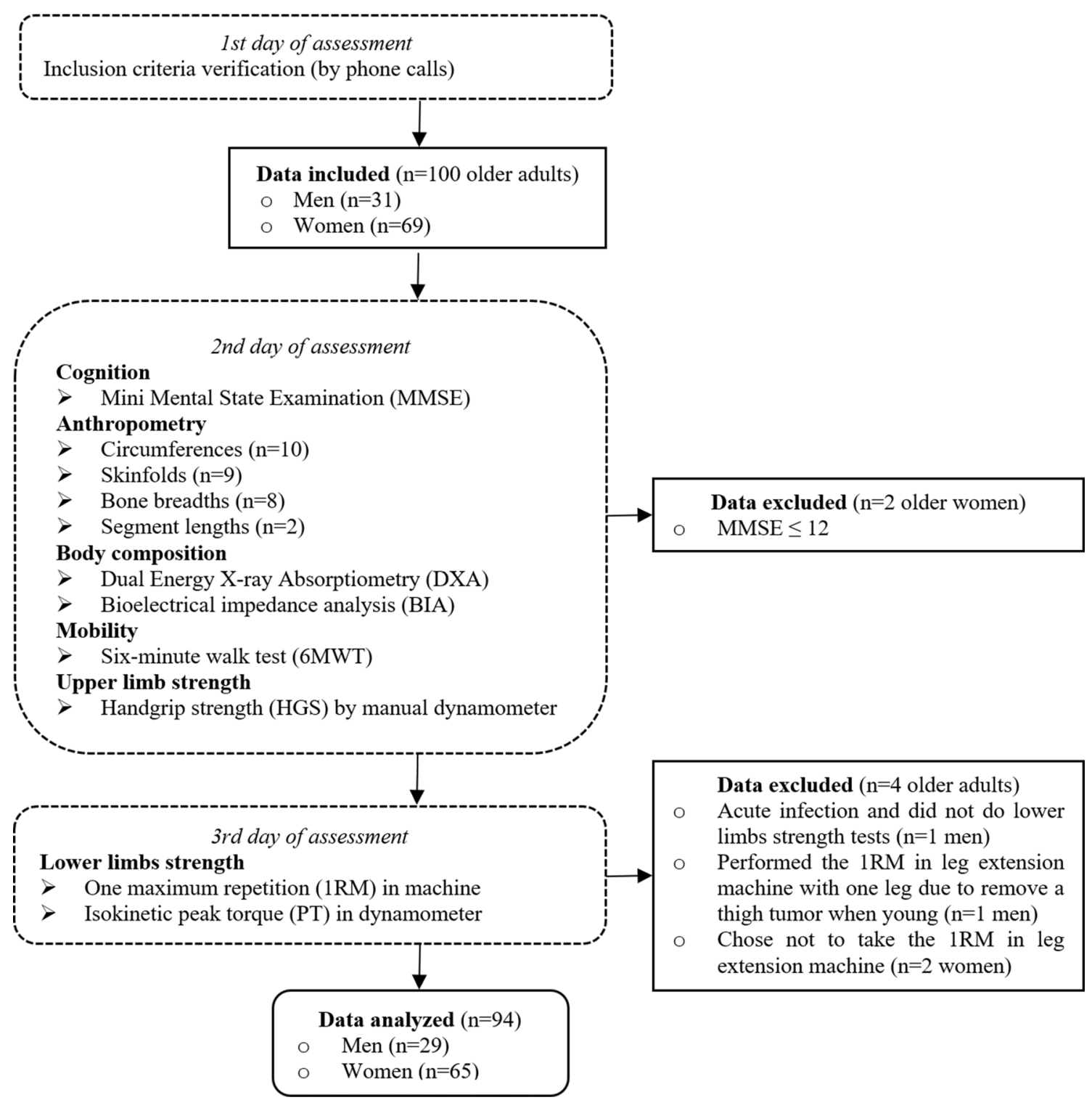

Fig. 1 Study phases and data from older adults included, excluded, analyzed and procedure flow

\section{Cognition assessment}

The validated Mini Mental State Examination (MMSE) was used to assess participants' cognition status and to ensure that participants understood the other tests executed in the present study [36]. The MMSE was executed in a quiet room, face to face with the researcher. Those who have MMSE $\leq 12$ were considered with dementia and were excluded [37].

\section{Measure of body-size variables}

Forty-nine body-size variables (Additional file 1: Supplement A) were collected to propose allometric exponents and to normalize performance in muscle strength tests.
The selection of these variables were based on those previously used to calculate body indexes [38-46], and involved anthropometric measurements [47] and body composition (Dual Energy X-ray Absorptiometry-DXA and bioelectrical impedance analysis-BIA), as briefly detailed below (body indexes).

Measures and instruments utilized were: body mass (Filizola ${ }^{\circledR}$ digital scale, model Personal, Brazil), height $\left(\right.$ Sanny ${ }^{\circledR}$ wall-mounted aluminum stadiometer, Professional model ES2020, Brazil), circumferences (Sanny ${ }^{\circledR}$ inelastic and inextensible measuring tape, Brazil), skinfolds (Lange scientific skinfold caliper, Cambridge Scientific Instruments, Cambridge, Maryland), bone 
breadths (Sanny ${ }^{\circledR}$ anthropometer and small sliding caliper, Brazil), and segment lengths $\left(\right.$ Sanny ${ }^{\circledR}$ segmometer, Brazil), lean soft tissue (LST) components, appendicular skeletal muscle mass (ASM) and FFM (DXA, Hologic $^{\circledR}$, model QDR4500W, software version 11.2, Bedford, MA), FFM [46] (Bioimpedance Imp DF50 Body Composition Analysis, ImpediMed ${ }^{\circledR}$, Brisbane, Queensland, Australia).

Anthropometry (body mass, height, circumferences, skinfold andbone breadths) was collected according to a standardized procedure published elsewhere [47]. DXA involved a full body scan performed (according to the manufacturer's recommended procedures) and interpreted always by the same technician. BIA exam was conducted in controlled temperature room $\left(23^{\circ} \mathrm{C}\right)$ with the older adults backed on a litter in comfortable position after rest for $10 \mathrm{~min}$ in supine position, without footwear and adornments (rings and earrings), with legs separated and opened hands. Older adults were previously oriented ( $24 \mathrm{~h}$ before the exam) to avoid the consumption of alcohol and caffeine (coffee, tea, chocolate), diuretic medication, intense physical activity and meal four hours before the exam.

\section{Body indexes}

The body indexes derived from anthropometry were body mass index (BMI, $\mathrm{kg} / \mathrm{m}^{2}$ ) [38], body mass"height [39], human body surface area $\left(\mathrm{SA}, \mathrm{m}^{2}\right)$ [40], absolute mid-arm muscle circumference (MAMC, $\mathrm{cm}$ ) [41], corrected arm muscle area (CAMA, $\mathrm{cm}$ ) [42], arm fat area $\left(\mathrm{AFA}, \mathrm{cm}^{2}\right)$ [43], FFM [44] and fat mass (obtained by body mass difference). The body indexes derived from body composition were LST of arms and legs, ASM, ASM/ height $(m)^{2}$ [45], FFM estimated from BIA [46] and DXA, when fat mass were estimated by body mass difference.

\section{Mobility measurement}

The cut-off points for muscle weakness were established based on the main outcome (mobility limitation). Mobility was verified based on the six-minute walk test (6MWT) carried out in a corridor 30-m length. Along this path, at every three meters there was a cone to help researcher to precisely identify the walked distance [31]. Participants were instructed to cover the longest distance walking as faster as they could during the six-minute time. Nevertheless, participants could slow down, interrupt the walking, and resume the test whenever desired, although time was not paused. Total walked distance was recorded and mobility limitation was characterized when the $6 \mathrm{MWT}<400 \mathrm{~m}[48]$.

\section{Muscle strength measurements}

Muscle strength was measured using HGS, one maximum repetition measurement for knee extensors (1RM) and isokinetic knee extension peak torque at a velocity of $60^{\circ} / \mathrm{s}(\mathrm{PT})$. The maximum HGS was measured with a manual dynamometer $\left(\right.$ Jamar $^{\circledR}$, model 5030J1) using a previously published protocol [49]. Three attempts were performed, one minute apart, with the dominant hand and the highest result was recorded in $\mathrm{kg}$ as HGS $[50,51]$. The 1RM was estimated in a leg extension machine (Lion Fitness ${ }^{\circledR}$ model LFS) with a submaximal repetition protocol: $1 \mathrm{RM}=$ weight lifted $/\left(1.0278-\left[0.0278^{*} \mathrm{n}^{\circ}\right.\right.$ of reps $\left.]\right)$ [52]. The detailed protocol was published elsewhere [26]. Briefly, a warm-up with lowest load was executed with 10 repetitions. After two-min resting, the load was doubled and eight repetitions were performed. After three-min resting, the test started and initial load was based on participants body mass ( $45 \%$ for women and $64 \%$ for men). The goal was to perform a maximum of 10 repetitions in three possible attempts (separate with three minutes intervals). Therefore, depending on older adults' muscle strength level, these initial loads could be increased or decreased to estimate 1RM. The PT of the right lower limb was recorded with the Biodex (model System 4 Pro) isokinetic dynamometer and results are in newton-meter $(\mathrm{Nm})$ according to standardized protocol [53]. Briefly, a warm-up with 10 submaximal repetitions in angular speed of $60^{\circ} / \mathrm{s}$ was performed. After three-min resting, the test was started with executing five maximum repetitions verbally encouraged by researchers without visual feedback. 1RM was executed prior to the PT, and the time interval between these tests was at least $30 \mathrm{~min}$.

\section{Physical activity level measurement}

The International Physical Activity Questionnaire-Short Version was used to get physical activity level [54]. Physical activity level was dichotomized into sedentary (0) and irregularly active, active or very active (1). These two categories were introduced in the models to provide allometric exponents.

\section{Muscle strength normalization procedures (ratio standard/ muscle quality and allometric scaling)}

HGS, 1RM and PT were considered in three different ways: 1) absolute (non-normalized); 2) ratio standard or muscle quality (muscle strength/body-size variable); and 3) allometrically adjusted (muscle strength/body-size variable ${ }^{\mathrm{b}}$.

Allometric exponents $\left(^{b}\right)$ were proposed only for bodysize variables that showed significant correlation (Pearson's correlation) with muscle strength. To generate the allometric exponents, muscle strength $(\mathrm{Y})$ and body-size 
variables $(\mathrm{X})$ were converted to natural logarithm $(\mathrm{ln})$ and the slope of regression line is allometric exponent $\left({ }^{b}\right)$, according to more detail previously published [24]. Therefore, allometric exponents were discarded when the interaction (ln body-size variable*age* sex*physical activity level) was significant or when there was multicollinearity in the linear regression (variance inflation factor [VIF] $>10$ ) [55].

We also consider other allometric exponents $\left({ }^{b}\right)$ of the literature, as described in Table 1.

In order to verify whether normalization removed the influence of body size on muscle strength, the correlation between normalized muscle strength and body-size variables (body mass, height and body-size used) should be negligible $(r \leq 30)$ [57].

\section{Statistical analysis}

We recorded and reviewed the data by double typing, followed by an exploratory analysis for error detection. We use parametric statistics for continuous variables considering the central limit theorem [58].

\section{Proposition of cut-off points for muscle weakness}

Absolute muscle strength and normalized by ratio standard/muscle quality or allometric scaling had their area under the curve (AUC) quantified by the ROC curve. The Youden index [59] selected the most appropriate cut-off points with the best relationship between sensitivity and specificity for the primary main outcome (functional limitation: 6MWD < 400) [48].

The cut-off points were considered adequate when they have AUC $\geq 0.70$ [60] simultaneously for both sexes $(p<0.05)$ and when the correlation between muscle

Table 1 Allometric exponents $\left({ }^{b}\right)$ proposed in previous studies

\begin{tabular}{ll}
\hline Authors & $\begin{array}{l}\text { Normalized muscle strength for } \\
\text { body-size variable }\end{array}$ \\
\hline Jaric [56] & General muscle strength/body mass ${ }^{0.67}$ \\
Foley et al. [23] & HGS/body mass ${ }^{0.40}$ \\
Pua [25] & HGS/body mass ${ }^{0.63}$ \\
Maranhão Neto et al. [24] & HGS/body mass \\
& HGS/height ${ }^{0.84}$ \\
Abdalla et al. [26] & 1RM/body mass \\
& 1RM/body mass \\
Davies and Dalsky [28] & PT/body mass \\
& PT/body mass \\
& PT/body mass \\
& PT/body mass* \\
&
\end{tabular}

HGS handgrip strength, 1RM one maximum repetition measurement for knee extensors, $P T$ isokinetic knee extension peak torque at $60 \%$ strength and body-size variables (body mass, height and body-size used) were negligible $(r \leq 0.30)$ [57].

For each muscle strength test (HGS, 1RM and PT), way (non-normalized, ratio standard/muscle quality and allometric scaling) and for each and sex was selected the adequate cut-off point according the superior accuracy. When there was a tie in accuracy, the variable with the greatest sensitivity or specificity was chosen. Finally, the AUC-ROC curves of non-normalized and normalized muscle strength were compared with each other to decide the best cut-off point.

The analyzes were performed using the SPSS 25.0 statistical package, and the ROC curves and Youden index in MedCalc 15.2 with a previously established level of significance $(\alpha=5 \%)$.

\section{Results}

Sample was encompassed by 100 older adults (69 women) who agreed to participate in the study. From those, 6 were excluded for different reasons, as the stages of the study proceeded, as detailed in Fig. 1. Therefore, the final sample comprised 29 older men (31\%) and 65 older women (69\%).

Sample characterization according to sex is shown in Table 2. About main outcome of the study, twenty-five women $(38.5 \%)$ and seven men $(24.1 \%)$ had functional limitation $(6 \mathrm{MWT}<400 \mathrm{~m})$.

The correlations between muscle strength and bodysize variables are also shown in Table 2. Most of the body-size variables showed a significant correlation with muscle strength $(\mathrm{r}=-0.41$ to $0.75 ; p<0.05)$. Non-significant correlations between body-size variables and muscle strength tests are shown in Additional file 1: Supplement B.

Allometric exponents were proposed for those bodysize variables that showed a significant relationship with muscle strength (Table 2). Linear regressions to obtain allometric exponents are shown in Additional file 1: Supplement $C$. All regressions were significant to explain muscle strength $(p<0.05)$, with adjusted $\mathrm{R}^{2}$ ranging from 0.39 to 0.61 . The regression coefficients $(\beta)$ obtained for each body-size variable represent the allometric exponents obtained. For HGS, the allometric exponents of triceps, pectoral, abdominal and thigh skinfolds were discarded because the interaction terms were statistically significant $(p<0.05)$ and have accentuated multicollinearity $(\mathrm{VIF}>10)$. The remaining allometric exponents were used to perform normalization (for example, 1RM/body mass $^{0.44}$ ).

The sex-specific cut-off points proposed for HGS, 1RM and PT (non-normalized, ratio standard/muscle quality and allometric scaling) to identify muscle weakness are presented in the Additional file 1: Supplement D. In 
Table 2 Descriptive analysis and significant correlations of muscle strength with body-size variables in older men and women ( $\mathrm{n}=94$ )

\begin{tabular}{|c|c|c|c|c|c|c|c|c|c|c|c|}
\hline \multirow[t]{3}{*}{ Variables } & \multicolumn{4}{|c|}{ Older Men $(n=29)$} & \multicolumn{4}{|c|}{ Older Women $(n=65)$} & \multicolumn{3}{|c|}{$\begin{array}{l}\text { Correlation (r) with Muscle } \\
\text { Strength }\end{array}$} \\
\hline & \multirow[t]{2}{*}{$M$} & \multicolumn{2}{|c|}{$95 \% \mathrm{Cl}$} & \multirow[t]{2}{*}{ SD } & \multirow[t]{2}{*}{ M } & \multicolumn{2}{|c|}{$95 \% \mathrm{Cl}$} & \multirow[t]{2}{*}{ SD } & \multirow[t]{2}{*}{ HGS (kg) } & \multicolumn{2}{|c|}{ Knee Extension } \\
\hline & & LL & UL & & & LL & UL & & & 1RM (kg) & PT (Nm) \\
\hline Age (years) & 71.2 & 68.5 & 73.9 & 7.1 & 69.7 & 68.2 & 71.2 & 6.1 & & & \\
\hline Mini-Mental State Examination (0-19) & 17.6 & 16.9 & 18.2 & 1.8 & 17.4 & 16.9 & 17.8 & 1.8 & & & \\
\hline \multicolumn{12}{|l|}{ Body-size variables } \\
\hline \multicolumn{12}{|l|}{ Anthropometry } \\
\hline Body mass (kg) & 73.0 & 67.7 & 78.3 & 13.9 & 66.9 & 64.0 & 69.8 & 11.6 & $0.37^{\dagger}$ & $0.39^{+}$ & $0.40^{\dagger}$ \\
\hline Height (m) & 1.7 & 1.6 & 1.7 & 0.1 & 1.6 & 1.5 & 1.6 & 0.1 & $0.71^{\dagger}$ & $0.62^{\dagger}$ & $0.68^{\dagger}$ \\
\hline \multicolumn{12}{|l|}{ Circumferences $(\mathrm{cm})$} \\
\hline Forearm & 26.0 & 25.2 & 26.8 & 2.0 & 23.8 & 23.3 & 24.4 & 2.2 & $0.50^{\dagger}$ & $0.40^{\dagger}$ & $0.46^{\dagger}$ \\
\hline Calf & 35.8 & 34.4 & 37.1 & 3.5 & 34.8 & 34.1 & 35.5 & 2.9 & $0.34^{*}$ & $0.37^{\dagger}$ & $0.37^{\dagger}$ \\
\hline Chest & 97.8 & 94.3 & 101.4 & 9.4 & 93.2 & 91.3 & 95.1 & 7.7 & $0.26^{*}$ & $0.32^{*}$ & $0.36^{\dagger}$ \\
\hline Waist & 92.1 & 87.8 & 96.5 & 11.4 & 86.5 & 84.0 & 89.0 & 10.0 & & $0.26^{*}$ & $0.30^{*}$ \\
\hline \multicolumn{12}{|l|}{ Skinfold thickness (mm) } \\
\hline Triceps & 15.2 & 12.9 & 17.5 & 6.0 & 25.8 & 24.1 & 27.4 & 6.7 & $-0.39^{\dagger}$ & $-0.22^{*}$ & $-0.25^{*}$ \\
\hline Biceps & 8.0 & 6.7 & 9.4 & 3.5 & 15.4 & 14.0 & 16.7 & 5.4 & $-0.40^{\dagger}$ & $-0.28^{*}$ & $-0.31^{*}$ \\
\hline Midaxillary & 18.5 & 15.5 & 21.5 & 7.8 & 23.9 & 22.2 & 25.6 & 6.9 & $-0.26^{*}$ & & \\
\hline Pectoral & 16.8 & 14.7 & 18.9 & 5.5 & 14.6 & 13.0 & 16.2 & 6.4 & $0.21^{*}$ & & \\
\hline Suprailiac & 19.7 & 15.8 & 23.5 & 10.1 & 29.7 & 27.8 & 31.7 & 7.8 & $-0.29 *$ & & \\
\hline Abdominal (vertical) & 26.2 & 23.1 & 29.3 & 8.1 & 33.7 & 31.5 & 35.9 & 8.8 & $-0.22^{*}$ & & \\
\hline Thigh (midline) & 17.9 & 15.0 & 20.8 & 7.6 & 32.1 & 29.5 & 34.8 & 10.7 & $-0.35^{*}$ & $-0.27^{*}$ & $-0.38^{\dagger}$ \\
\hline Medial calf & 11.8 & 9.3 & 14.3 & 6.6 & 23.8 & 21.9 & 25.7 & 7.6 & $-0.41^{\dagger}$ & $-0.28^{*}$ & $-0.33^{*}$ \\
\hline \multicolumn{12}{|l|}{ Bone breadths (mm) } \\
\hline Biacromial & 39.9 & 38.9 & 41.0 & 2.8 & 37.1 & 36.6 & 37.6 & 2.1 & $0.63^{\dagger}$ & $0.58^{\dagger}$ & $0.60^{+}$ \\
\hline Bitrochanteric & 33.7 & 33.1 & 34.3 & 1.7 & 33.4 & 32.8 & 34.0 & 2.3 & & $0.20^{*}$ & $0.22^{*}$ \\
\hline Ankle (bimalleolar) & 7.0 & 6.8 & 7.2 & 0.5 & 6.3 & 6.2 & 6.4 & 0.4 & $0.59^{\dagger}$ & $0.47^{\dagger}$ & $0.52^{+}$ \\
\hline Elbow & 6.7 & 6.5 & 6.9 & 0.5 & 5.8 & 5.7 & 6.0 & 0.5 & $0.53^{+}$ & $0.40^{\dagger}$ & $0.44^{\dagger}$ \\
\hline Wrist & 5.7 & 5.6 & 5.9 & 0.4 & 5.1 & 5.0 & 5.1 & 0.4 & $0.52^{\dagger}$ & $0.37^{\dagger}$ & $0.44^{\dagger}$ \\
\hline Chest & 30.9 & 29.9 & 31.9 & 2.6 & 27.8 & 27.4 & 28.3 & 1.8 & $0.58^{\dagger}$ & $0.49^{\dagger}$ & $0.59^{\dagger}$ \\
\hline \multicolumn{12}{|l|}{ Segment lenghts $(\mathrm{cm})$} \\
\hline Knee height & 53.5 & 52.4 & 54.5 & 2.7 & 49.5 & 49.0 & 50.0 & 2.1 & $0.62^{+}$ & $0.55^{\dagger}$ & $0.58^{\dagger}$ \\
\hline Half arm span & 87.3 & 85.5 & 89.2 & 4.8 & 80.8 & 79.8 & 81.7 & 3.8 & $0.71^{+}$ & $0.62^{+}$ & $0.58^{+}$ \\
\hline \multicolumn{12}{|l|}{ Body indexes } \\
\hline Derived from anthropometry & & & & & & & & & & & \\
\hline Body mass*height $\left(\mathrm{kg}^{*} \mathrm{~m}\right)$ & 123.3 & 112.6 & 134.1 & 28.2 & 104.7 & 99.8 & 109.7 & 20.0 & $0.49^{\dagger}$ & $0.49^{\dagger}$ & $0.52^{+}$ \\
\hline $\mathrm{SA}\left(\mathrm{m}^{2}\right)$ & 1.9 & 1.8 & 1.9 & 0.2 & 1.7 & 1.7 & 1.8 & 0.2 & $0.47^{\dagger}$ & $0.48^{\dagger}$ & $0.50^{+}$ \\
\hline MAMC (cm) & 24.2 & 23.0 & 25.3 & 2.9 & 21.9 & 21.3 & 22.5 & 2.6 & $0.45^{\dagger}$ & $0.39^{\dagger}$ & $0.45^{\dagger}$ \\
\hline CAMA $\left(\mathrm{cm}^{2}\right)$ & 37.1 & 32.7 & 41.6 & 11.7 & 32.2 & 29.9 & 34.5 & 9.2 & $0.37^{\dagger}$ & $0.33^{\dagger}$ & $0.40^{\dagger}$ \\
\hline AFA $\left(\mathrm{cm}^{2}\right)$ & 16.3 & 14.0 & 18.6 & 6.0 & 22.9 & 21.3 & 24.6 & 6.6 & $-0.23^{*}$ & & \\
\hline $\mathrm{FFM}_{(\text {LEAN et al. [44]]) }}(\mathrm{kg})$ & 52.1 & 49.6 & 54.6 & 6.6 & 37.1 & 36.0 & 38.2 & 4.6 & $0.75^{+}$ & $0.66^{\dagger}$ & $0.67^{\dagger}$ \\
\hline Fat mass $($ LEAN et al. [44]] $(\mathrm{kg})$ & 20.9 & 17.7 & 24.0 & 8.2 & 29.8 & 27.9 & 31.7 & 7.8 & $-0.22^{*}$ & & \\
\hline Derived from body composition & & & & & & & & & & & \\
\hline Left arm LST (kg) & 2.4 & 2.1 & 2.6 & 0.6 & 1.5 & 1.4 & 1.6 & 0.3 & $0.72^{+}$ & $0.62^{+}$ & $0.66^{\dagger}$ \\
\hline Right arm LST (kg) & 2.8 & 2.5 & 3.0 & 0.6 & 1.8 & 1.7 & 1.9 & 0.4 & $0.72^{+}$ & $0.61^{\dagger}$ & $0.60^{\dagger}$ \\
\hline Left leg LST (kg) & 7.7 & 7.1 & 8.3 & 1.6 & 5.5 & 5.3 & 5.8 & 1.0 & $0.67^{\dagger}$ & $0.64^{\dagger}$ & $0.64^{+}$ \\
\hline Right leg LST (kg) & 8.0 & 7.4 & 8.6 & 1.6 & 5.7 & 5.4 & 5.9 & 1.0 & $0.70^{+}$ & $0.64^{+}$ & $0.65^{\dagger}$ \\
\hline Arms LST (kg) & 5.1 & 4.7 & 5.6 & 1.2 & 3.3 & 3.2 & 3.5 & 0.7 & $0.74^{\dagger}$ & $0.63^{\dagger}$ & $0.64^{\dagger}$ \\
\hline Legs LST (kg) & 15.7 & 14.6 & 16.9 & 3.1 & 11.2 & 10.7 & 11.6 & 1.9 & $0.69^{\dagger}$ & $0.65^{\dagger}$ & $0.66^{\dagger}$ \\
\hline
\end{tabular}


Table 2 (continued)

\begin{tabular}{|c|c|c|c|c|c|c|c|c|c|c|c|}
\hline \multirow[t]{3}{*}{ Variables } & \multicolumn{4}{|c|}{ Older Men $(n=29)$} & \multicolumn{4}{|c|}{ Older Women $(n=65)$} & \multicolumn{3}{|c|}{$\begin{array}{l}\text { Correlation (r) with Muscle } \\
\text { Strength }\end{array}$} \\
\hline & \multirow[t]{2}{*}{ M } & \multicolumn{2}{|c|}{$95 \% \mathrm{Cl}$} & \multirow[t]{2}{*}{ SD } & \multirow[t]{2}{*}{ M } & \multicolumn{2}{|c|}{$95 \% \mathrm{Cl}$} & \multirow[t]{2}{*}{ SD } & \multirow[t]{2}{*}{ HGS (kg) } & \multicolumn{2}{|c|}{ Knee Extension } \\
\hline & & LL & UL & & & LL & UL & & & 1RM (kg) & PT (Nm) \\
\hline ASM (kg) & 20.9 & 19.3 & 22.5 & 4.2 & 14.5 & 13.9 & 15.1 & 2.5 & $0.72^{+}$ & $0.65^{\dagger}$ & $0.66^{+}$ \\
\hline ASM/height ${ }^{2}\left(\mathrm{~kg} / \mathrm{m}^{2}\right)$ & 7.3 & 7.0 & 7.7 & 1.0 & 6.0 & 5.7 & 6.2 & 0.9 & $0.55^{\dagger}$ & $0.51^{\dagger}$ & $0.48^{\dagger}$ \\
\hline FFM $_{\text {(Baumgartner et al. [46]) }}(\mathrm{kg})$ & 54.3 & 51.3 & 57.3 & 7.7 & 45.5 & 44.0 & 47.0 & 6.1 & $0.60^{\dagger}$ & $0.55^{\dagger}$ & $0.57^{\dagger}$ \\
\hline $\mathrm{FFM}_{(\mathrm{DXA})}(\mathrm{kg})$ & 51.5 & 47.9 & 55.0 & 9.4 & 38.8 & 37.4 & 40.3 & 5.8 & $0.68^{+}$ & $0.59^{+}$ & $0.61^{\dagger}$ \\
\hline Fat mass $_{(\mathrm{DXA})}(\mathrm{kg})$ & 21.5 & 18.8 & 24.2 & 7.1 & 28.1 & 26.3 & 29.9 & 7.2 & $-0.20^{*}$ & & \\
\hline \multicolumn{12}{|l|}{ Mobility } \\
\hline Six-minute walk test (6MWT) & 464.7 & 431.1 & 498.3 & 88.3 & 412.7 & 389.9 & 435.5 & 92.0 & & & \\
\hline Functional limitation (6MWT<400 m); \% & $24.1 \%$ & & & & $38.5 \%$ & & & & & & \\
\hline \multicolumn{12}{|l|}{ Muscle strength } \\
\hline HGS (kg) & 36.4 & 33.1 & 39.7 & 8.6 & 24.1 & 23.0 & 25.2 & 4.5 & & & \\
\hline $1 \mathrm{RM}(\mathrm{kg})$ & 66.8 & 56.9 & 76.7 & 26.0 & 40.8 & 36.9 & 44.8 & 15.9 & & & \\
\hline No. of reps to estimate 1RM & 7.2 & 6.3 & 8.1 & 2.3 & 6.6 & 6.0 & 7.2 & 2.4 & & & \\
\hline PT (Nm) & 119.8 & 102.4 & 137.2 & 45.6 & 73.2 & 66.8 & 79.6 & 25.9 & & & \\
\hline
\end{tabular}

$M$ mean, $C l$ confidence interval, $L L$ lower limit, UL upper limit, SD standard deviation, HGS handgrip strength, 1RM one maximum repetition measurement for knee extensors, $P T$ isokinetic knee extension peak torque at $60 \% \mathrm{~s}, \mathrm{Nm}$ Newton meter, $S A$ human body surface area, $M A M C$ mid-arm muscle circumference, $C A M A$ corrected arm muscle area, AFA arm fat area, FFM fat-free mass, LST lean soft tissue, ASM appendicular skeletal muscle mass, DXA Dual-energy X-ray absorptiometry

${ }^{*} p<0.05$ and ${ }^{\dagger} p<0.001$ (statistically significant correlation)

the same supplement there are also presented correlations between muscle strength and body size (body mass, height and body-size variable used in normalization).

Non-normalized HGS, 1RM and PT cut-off points to identify muscle weakness were not adequate for both sexes or because they did not present $A U C \geq 0.70$ $(p<0.05)$ or because they had a significant association with body size $(\mathrm{r}>0.30 ; p<0.05)$ (Additional file 1: Supplement D).

Table 3 shows the cut-off points based on the ratio standard/muscle quality and allometric scaling classified as adequate.

A comparison of the most accurate ROC curves is presented in Fig. 2 to support the decision for the best cut-off point between non-normalized, ratio standard/ muscle quality and allometric scaling of HGS and lower limbs strength (1RM and PT) for each sex.

For men, there were no differences in accuracy (AUC) to identify functional limitation between absolute muscle strength, normalized by ratio standard/muscle quality or by allometric scaling ( $p>0.05$; Fig. $2 \mathrm{a}-\mathrm{c})$. However, the absolute muscle strengths (HGS, 1RM and PT) previously indicated great dependence $(r>0.30)$ on body size (Additional file 1: Supplement D), suggesting the need for normalization to avoid errors in the classification of weakness. The normalized muscle strength increased the AUC and made it possible to classify muscle weakness of older adults with extreme body sizes, independently.
For women, only after normalizing muscle strength the AUC values perform acceptable to identify functional limitation (AUC >0.70; Fig. 2d, e). The exception was PT, when the absolute values already had adequate accuracy (AUC > 0.70), although without the desirable independence of body size. All the normalizations increased $\left(^{\ddagger}\right)$ the AUC $(p<0.001)$.

\section{Discussion}

Cut-off points based on upper and lower limbs muscle strength were proposed to identify muscle weakness in older adults of both sexes. The non-normalized cut-off points for HGS and lower limbs strength were significantly associated with body size, which involves biases to assess older adults with extreme body size (e.g., heavy or short). After normalizing HGS and lower limbs strength by the ratio standard/muscle quality or by the allometry, the association with body size was no longer relevant. In addition, for women, the accuracy to predict mobility limitation/muscle weakness from normalized muscle strength cut-off points become acceptable when compared to non-normalized strategy. In men, muscle strength normalization did not increase accuracy. However, all normalized models of both sexes avoided biases in the assessment of muscle weakness/mobility limitation, to isolate the natural interdependence between muscle strength and body size [24]. 
Table 3 Adequate cut-off points (AUC $\geq 0.70$ simultaneously in both sexes and $r \leq 0.30$ with body size) of handgrip strength (HGS), one maximum repetition measurement for knee extensors (1RM) and isokinetic knee extension peak torque at $60 \% \mathrm{~s}$ (PT) to identify muscle weakness

\begin{tabular}{|c|c|c|c|c|c|c|c|c|c|}
\hline \multirow[t]{2}{*}{ Variable } & \multirow[t]{2}{*}{ Unit } & \multicolumn{4}{|c|}{ Men $(n=29)$} & \multicolumn{4}{|c|}{ Women $(n=65)$} \\
\hline & & AUC & Cut-off point $(\leq)$ & Sens (\%) & Spe (\%) & AUC & Cut-off point $(\leq)$ & Sens (\%) & Spe $(\%)$ \\
\hline \multicolumn{10}{|l|}{$H G S(k g)$} \\
\hline /Forearm circumference & $(\mathrm{cm})$ & $0.74^{*}$ & 1.33 & 86 & 59 & $0.70^{*}$ & 1.04 & 84 & 58 \\
\hline \multicolumn{10}{|l|}{$1 R M(k g)$} \\
\hline /Body mass & $(\mathrm{kg})$ & $0.77^{*}$ & 0.85 & 86 & 68 & $0.76^{+}$ & 0.54 & 68 & 78 \\
\hline /Forearm circumference & $(\mathrm{cm})$ & $0.75^{*}$ & 2.16 & 86 & 77 & $0.70^{*}$ & 1.38 & 60 & 75 \\
\hline /Calf circumference & & $0.74^{*}$ & 1.65 & 86 & 77 & $0.70^{*}$ & 1.06 & 72 & 68 \\
\hline /Chest circumference & & $0.76^{*}$ & 0.64 & 86 & 73 & $0.71^{*}$ & 0.4 & 72 & 65 \\
\hline Waist circumference & & $0.78^{*}$ & 0.73 & 100 & 59 & $0.72^{*}$ & 0.37 & 60 & 80 \\
\hline /Triceps skinfold & $(\mathrm{mm})$ & $0.81^{\dagger}$ & 4.22 & 86 & 68 & $0.70^{*}$ & 1.40 & 60 & 75 \\
\hline /Bitrochanteric breadth & & $0.73^{*}$ & 1.72 & 86 & 77 & $0.70^{*}$ & 1.16 & 76 & 60 \\
\hline /Bimalleolar breadth & & $0.73^{*}$ & 8.77 & 86 & 77 & $0.70^{*}$ & 5.77 & 76 & 65 \\
\hline /Elbow breadth & & $0.73^{*}$ & 9.36 & 86 & 73 & $0.70^{*}$ & 6.57 & 76 & 63 \\
\hline ISA & $\left(m^{2}\right)$ & $0.75^{*}$ & 31.6 & 86 & 77 & $0.72^{*}$ & 21.2 & 72 & 68 \\
\hline /MAMC & $(\mathrm{cm})$ & $0.77^{*}$ & 2.42 & 86 & 73 & $0.72^{*}$ & 1.54 & 64 & 75 \\
\hline /CAMA & $\left(\mathrm{cm}^{2}\right)$ & $0.71^{*}$ & 2.03 & 100 & 41 & $0.73^{*}$ & 0.90 & 52 & 93 \\
\hline /FFM (Lean et al. [44]) & $(\mathrm{kg})$ & $0.76^{*}$ & 1.11 & 86 & 77 & $0.72^{*}$ & 1.00 & 72 & 68 \\
\hline /FFM (Baumgartner et al. [46]) & $(\mathrm{kg})$ & $0.78^{*}$ & 1.13 & 83 & 73 & $0.75^{\dagger}$ & 0.83 & 76 & 64 \\
\hline /Body mass 0.44 & $(\mathrm{~kg})$ & $0.75^{*}$ & 9.04 & 86 & 77 & $0.71^{*}$ & 6.03 & 76 & 63 \\
\hline /Body mass ${ }^{0,67}$ (Jaric [56]) & & $0.78^{*}$ & 3.40 & 86 & 77 & $0.73^{+}$ & 2.28 & 76 & 65 \\
\hline /Body mass ${ }^{0.96}$ (Abdalla et al. [53]) & & $0.77^{*}$ & 1.00 & 86 & 68 & $0.75^{\dagger}$ & 0.45 & 44 & 100 \\
\hline /Body mass 0.69 (Abdalla et al. [53]) & & $0.78^{*}$ & 3.06 & 86 & 77 & $0.73^{*}$ & 1.48 & 44 & 98 \\
\hline /Calf circumference ${ }^{1.10}$ & $(\mathrm{~cm})$ & $0.75^{*}$ & 1.14 & 86 & 77 & $0.71^{*}$ & 0.70 & 68 & 73 \\
\hline /Bimalleolar breadth 1.20 & $(\mathrm{~mm})$ & $0.71^{*}$ & 6.01 & 86 & 68 & $0.70^{*}$ & 3.93 & 76 & 65 \\
\hline$/(\text { Body mass*height })^{0.48}$ & $\left(\mathrm{~kg}^{*} \mathrm{~m}\right)$ & $0.75^{*}$ & 5.83 & 86 & 77 & $0.72^{*}$ & 4.06 & 76 & 65 \\
\hline$/ \mathrm{SA}^{0.93}$ & $\left(m^{2}\right)$ & $0.75^{*}$ & 33 & 86 & 77 & $0.72^{*}$ & 22.7 & 76 & 65 \\
\hline$/$ FFM $^{0.88}$ (Lean et al. [44]) & $(\mathrm{kg})$ & $0.75^{*}$ & 1.77 & 86 & 77 & $0.71^{*}$ & 1.53 & 76 & 65 \\
\hline /FFM".067 (Baumgartner et al. [46]) & & $0.76^{*}$ & 3.94 & 83 & 77 & $0.72^{*}$ & 2.91 & 76 & 64 \\
\hline \multicolumn{10}{|l|}{ PT $(\mathrm{Nm})$} \\
\hline /Height & (m) & $0.93^{+}$ & 54.1 & 86 & 95 & $0.74^{+}$ & 44.1 & 64 & 75 \\
\hline /Knee height & $(\mathrm{cm})$ & $0.94^{+}$ & 1.83 & 100 & 86 & $0.75^{\dagger}$ & 1.44 & 68 & 73 \\
\hline ISA & $\left(m^{2}\right)$ & $0.94^{+}$ & 56.9 & 100 & 82 & $0.81^{\dagger}$ & 36.3 & 64 & 88 \\
\hline$/ F_{\text {(Lean et al. [44]) }}$ & $(\mathrm{kg})$ & $0.93^{+}$ & 1.79 & 86 & 95 & $0.81^{\dagger}$ & 1.84 & 76 & 80 \\
\hline /Left leg LST & (g) & $0.82^{+}$ & 0.015 & 100 & 55 & $0.76^{\dagger}$ & 0.012 & 68 & 80 \\
\hline /Right leg LST & & $0.77^{*}$ & 0.015 & 100 & 50 & $0.78^{\dagger}$ & 0.013 & 76 & 78 \\
\hline /Legs LST & & $0.81^{*}$ & 0.0063 & 71 & 82 & $0.77^{\dagger}$ & 0.0065 & 76 & 78 \\
\hline /ASM & $(\mathrm{kg})$ & $0.81^{*}$ & 4.66 & 71 & 86 & $0.77^{\dagger}$ & 5.01 & 76 & 78 \\
\hline$/ F_{\text {(Baumgartner et al. [46]) }}$ & & $0.93^{+}$ & 1.63 & 83 & 95 & $0.85^{\dagger}$ & 1.60 & 88 & 77 \\
\hline$/ \mathrm{FFM}_{(\mathrm{DXA})}$ & & $0.84^{+}$ & 2.08 & 100 & 59 & $0.79^{\dagger}$ & 1.89 & 76 & 75 \\
\hline /Body mass ${ }^{0.67}$ (Davies and Dalsky [28]) & $(\mathrm{kg})$ & $0.93^{+}$ & 5.06 & 86 & 95 & $0.82^{\dagger}$ & 3.71 & 68 & 88 \\
\hline /Body mass $^{0.72(\text { Davies and Dalsky [28]) }}$ & & $0.94^{+}$ & 4.1 & 86 & 95 & $0.82^{+}$ & 3.14 & 72 & 85 \\
\hline /Body mass ${ }^{0.74(\text { Davies and Dalsky [28]) }}$ & & $0.93^{+}$ & 3.77 & 86 & 95 & $0.82^{\dagger}$ & 2.87 & 72 & 85 \\
\hline /Body mass ${ }^{0.67}$ (Jaric [56]) & & $0.93^{+}$ & 5.06 & 86 & 95 & $0.82^{+}$ & 3.71 & 68 & 88 \\
\hline /Height 3.27 & (m) & $0.86^{+}$ & 19.2 & 100 & 77 & $0.74^{+}$ & 17.4 & 72 & 65 \\
\hline /Knee height ${ }^{1.82}$ & $(\mathrm{~cm})$ & $0.90^{+}$ & 0.068 & 100 & 86 & $0.74^{+}$ & 0.053 & 64 & 75 \\
\hline /Half arm span 1.62 & & $0.88^{+}$ & 0.076 & 100 & 73 & $0.75^{\dagger}$ & 0.066 & 84 & 58 \\
\hline /Biacromial breadth2.15 & $(\mathrm{mm})$ & $0.82^{+}$ & 0.038 & 86 & 68 & $0.77^{\dagger}$ & 0.03 & 76 & 73 \\
\hline
\end{tabular}


Table 3 (continued)

\begin{tabular}{|c|c|c|c|c|c|c|c|c|c|}
\hline \multirow[t]{2}{*}{ Variable } & \multirow[t]{2}{*}{ Unit } & \multicolumn{4}{|c|}{ Men $(n=29)$} & \multicolumn{4}{|c|}{ Women $(n=65)$} \\
\hline & & AUC & Cut-off point $(\leq)$ & Sens (\%) & Spe (\%) & AUC & Cut-off point $(\leq)$ & Sens (\%) & Spe (\%) \\
\hline /Bimalleolar breadth ${ }^{1.54}$ & & $0.81^{*}$ & 5.04 & 86 & 73 & $0.80^{+}$ & 4.64 & 92 & 60 \\
\hline$/(\text { Body mass*height })^{0.43}$ & $\left(\mathrm{~kg}^{*} \mathrm{~m}\right)$ & $0.94^{+}$ & 13 & 100 & 82 & $0.80^{\dagger}$ & 10.5 & 84 & 65 \\
\hline$/ S A^{0.83}$ & $\left(m^{2}\right)$ & $0.94^{+}$ & 53.8 & 86 & 95 & $0.80^{+}$ & 50 & 84 & 65 \\
\hline /Left leg LST $T^{0.43}$ & (g) & $0.92^{+}$ & 2.26 & 100 & 77 & $0.77^{+}$ & 1.83 & 76 & 70 \\
\hline /Right leg LST 0.48 & & $0.90^{+}$ & 1.39 & 100 & 73 & $0.78^{+}$ & 1.16 & 80 & 70 \\
\hline /Legs LST.47 & & $0.92^{+}$ & 1.07 & 100 & 73 & $0.77^{+}$ & 0.87 & 76 & 70 \\
\hline
\end{tabular}

Dependent variable (primary outcome): functional limitation (6MWT $<400 \mathrm{~m}$ )

$A U C$ area under the curve, $p$ significance, Sens sensibility, Spe specificity, SA human body surface area, MAMC mid-arm muscle circumference, CAMA corrected arm muscle area, FFM Fat-free mass, LST lean soft tissue, ASM appendicular skeletal muscle mass, DXA Dual-energy X-ray absorptiometry, $6 M W T$ six-minute walk test ${ }^{*} p<0.05$ and ${ }^{\dagger} p<0.001$ (statistically significant AUC)

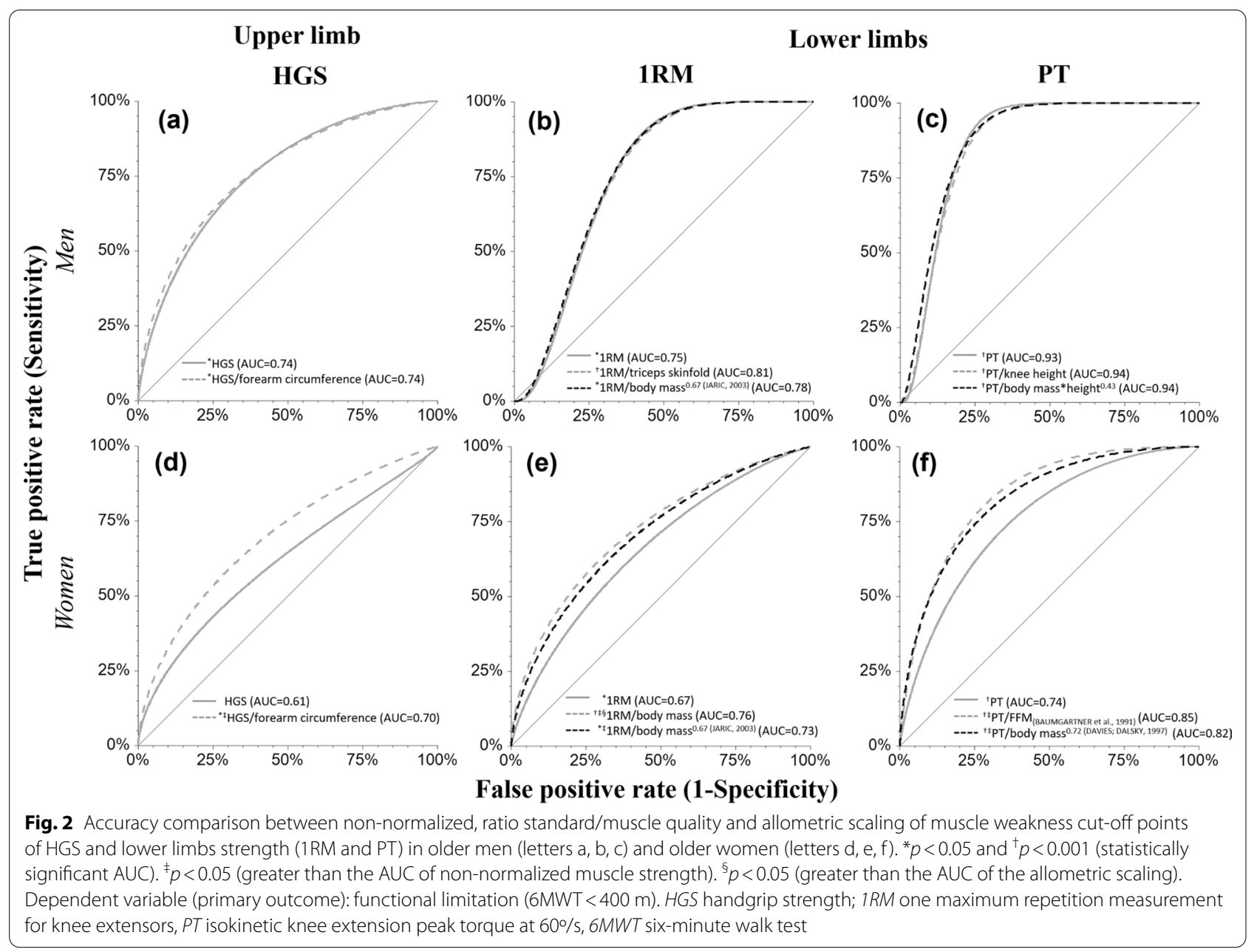

To the best of our knowledge, this is the first study to propose muscle weakness cut-off points for the HGS and PT allometrically adjusted in older adults. In a previous study, 1RM was allometrically adjusted for body mass [26], but not according to all other potential body size variables. Indeed, we expanded the number of variables that can be used to normalize 1RM with allometry $(n=8)$ in order to augment model's accuracy for identifying muscle weakness regardless of extreme body sizes. Other studies proposed muscle weakness cut-off points with 
HGS normalized by ratio standard (body mass or BMI) $[18,61,62]$ or stratified by BMI quartiles [7]. There are also muscle weakness cut-off points for PT normalized by body mass [19]. However, these studies did not compare the accuracy of normalized with non-normalized muscle strength to identify muscle weakness. Furthermore, they did not explore other body-size variables to normalize muscle strength.

Previous studies have proposed allometric exponents to normalize muscle strength, including HGS [23-25], $1 \mathrm{RM}$ [26], PT [28, 39], and they are comparable with the ones found in the present study. Curvilinear (allometric) relationship variables is confirmed when allometric coefficient $\left({ }^{b}\right)$ is between 0.00 and 0.99 [63], while the linear relationship is characterized when the exponent is $\geq 1.00$ [63]. In the literature, body mass generally presents an allometric relationship with muscle strength independently of the test (HGS, 1RM or PT; Table 1), confirming our findings (Additional file 1: Supplement C), when b exponents were 0.22 (HGS), 0.44 (1RM) and 0.37 (PT). Contrarily, height tends to have a linear relationship $\left({ }^{b} \geq 1.00\right)$ with muscle strength [24], what was also confirmed by our proposed allometric exponents (Additional file 1: Supplement C), that were between 1.87 and 3.27.

Some strengths of our study are noteworthy. We proposed muscle weakness cut-off points for isokinetic dynamometer, considered as a "gold standard" resource to assess lower limbs strength. The estimated 1RM obtained with submaximal repetition protocol and the HGS are valid for older adults, even for those with muscle weakness [53,64]. An extensive number of body-size variables $(n=49)$ were tested in our study, expanding the possibilities to promote the normalization of performance in muscle strength tests. Furthermore, regardless of the chosen muscle strength test to evaluate weakness, our findings can be applied with sufficient accuracy (AUC $>0.70$ ) both for scientific research $(\mathrm{PT})$ and population-based monitoring (HGS and 1RM). Nevertheless, this study is not without limitations. The individual muscle strength decline along aging may have been underestimated with the cross-sectional design. The small and local sample size of our study, requiring caution to extrapolate these findings inferentially to other populations. Another limitation is the utilization of open kinetic chain test in the case of $1 \mathrm{RM}$ in a leg extension machine, a movement far to the natural comportment during daily living. Our suggestion for future studies is to establish allometric exponents and cut-off points for a close kinetic chain exercise like leg press or squat, which require movements more closely associated with daily live.

We found greater accuracy (AUC) for normalized lower limbs strength (isokinetic dynamometer and leg extension machine) than manual dynamometer (normalized upper extremity strength), usually adopted to predict mobility limitations/muscle weakness [8]. However, the isokinetic dynamometer is expensive and generally more available in terms of research. Even though, our normalized models are also applicable in clinical practice from manual dynamometers (widely available in geriatric environments) and leg extension machines (available in most fitness centers, adequate environment for intervention against aged-related muscle weakness) [35]. The assessment of HGS and 1RM and proper classification of muscle weakness amongst older adults should be frequent in clinical practice to better target health expending, avoiding unnecessary expenditures. Future research should observe if proposed allometric exponents can be utilized to normalize muscle strength for different older adults' population, with other ethnicity/race characteristics.

As an applied example to avoid false positive diagnosis for muscle weakness, we hypothesize one older man with extreme lower values of body mass (42 kg), $1.57 \mathrm{~m}$ of height, who performed PT of $85.2 \mathrm{Nm}$. If we consider our absolute cut-off point $(\leq 85.4 \mathrm{Nm})$, this older man has muscle weakness confirmed. However, when considered the normalized PT/([body mass"height $\left.]^{0.43}\right)$, the adjusted value $(14.1 \mathrm{Nm} / \mathrm{kg} / \mathrm{m})$ is above of the cut-off point $(13.0$ $\mathrm{Nm} / \mathrm{kg}{ }^{*} \mathrm{~m}$; Table 3). Normalization would also avoid false negative cases, for large body size of older adults. For example, if an older woman with $90 \mathrm{~kg}$ performs $1 \mathrm{RM}$ of $38.2 \mathrm{~kg}$ and considering our absolute cut-off point $(\leq 38.1 \mathrm{~kg})$, this older woman does not have weakness. However, when considered the normalized (1RM/ body mass $\left.{ }^{0.67}\right)$, the adjusted value $(1.87 \mathrm{~kg} / \mathrm{kg})$ is below of the cut-off point $(2.28 \mathrm{~kg} / \mathrm{kg}$; Table 3$)$, characterizing weakness and a false negative case if non-normalized cut-off point were considered. The mistaken framing of false weakness cases could greatly impact the financial resources in the health and older people care systems. Especially in low- and middle-income countries, where these resources are scarcer.

\section{Conclusion}

Upper and lower limbs muscle weakness cut-off points standardized according to body size were proposed for older adults of both sexes. The normalization has increased accuracy for identify women with muscle weakness; but not in men, whose absolute muscle strength values have an acceptable accuracy. However, normalization made muscle strength independent of body size, confirming our hypothesis and preventing bias in the evaluation of older adults with extreme body size (e.g., very low or very heavy). Forty-nine valid models were proposed for older adults of both sexes, with different possibilities of body's normalization of 
muscle strength, which broadens the interpretation of muscle strength with less risk of attributing a false-negative/positive diagnosis to muscle weakness.

\author{
Abbreviations \\ b: Allometric exponent; HGS: Handgrip strength; PT: Isokinetic knee extension \\ peak torque at $60^{\circ} / \mathrm{s} ; 1 \mathrm{RM}$ : One maximum repetition measurement for knee \\ extensors; 6MWT: Six-minute walk test.
}

\section{Supplementary Information}

The online version contains supplementary material available at https://doi. org/10.1186/s13102-021-00390-1.

Additional file 1. SUPPLEMENT A - Body size variables $(n=49)$ to normalize muscle strength. SUPPLEMENT B - Non-significant correlations between body-size variables and muscle strength tests. SUPPLEMENT C - Linear regressions to obtain allometric exponents for handgrip strength (HGS), one maximum repetition measurement for knee extensors (1RM) and isokinetic knee extension peak torque at $60 \% \mathrm{~s}$ (PT) in older men and women $(n=94)$. SUPPLEMENT D - Cut-off points to identify muscle weakness in older adults of the handgrip strength (HGS), one maximum repetition measurement for knee extensors (1RM) and isokinetic knee extension peak torque at $60 \%$ (PT) (non-normalized, ratio standard/muscle quality and allometric scaling), and the correlation of muscle strength with body size.

\section{Acknowledgements}

Not applicable.

\section{Authors' contributions}

PPA: Conceptualization; Roles/Writing —original draft; JM: Data curation, Visualization; LB: Methodology, PPA: Formal analysis; DRLM: Software, JM: Funding acquisition, Supervision; APS, ACRV and ASC: Investigation, LB, DMG, LSLS and MFTJ: Writing — review and editing; DRLM: Validation; and JM: Project administration, Resources. All authors have read and approved the manuscript.

\section{Funding}

This work was supported by the Conselho Nacional de Desenvolvimento Científico e Tecnológico (CNPq) [through PhD scholarship number 142248/2018-5 granted to Pedro Pugliesi Abdalla]; and the Coordenação de Aperfeiçoamento de Pessoal de Nível Superior (CAPES) [finance Code 001, through PhD scholarship number 88887.593242/2020-00 granted to Pedro Pugliesi Abdalla, and through scholarship number 88887.371516/2019-00 granted to Dalmo Roberto Lopes Machado]. The funding body had no role in the design of the study and collection, analysis, and interpretation of data and in writing the manuscript.

\section{Availability of data and materials}

The datasets generated during and/or analysed during the current study are available from the corresponding author on reasonable request.

\section{Declarations}

\section{Ethics approval and consent to participate}

The study was approved by the HC-FMRP-USP institutional review board (CAAE: 54345016.6.3001.5440) and written informed consent was obtained from all subjects. All methods were carried out in accordance with Declaration Helsinki.

\section{Consent for publication}

Not applicable.

\section{Competing interests}

The authors declare that they have no competing interests.

\section{Author details}

${ }^{1}$ College of Nursing at Ribeirão Preto, University of São Paulo, Ribeirão Preto, SP, Brazil. ${ }^{2}$ Faculty of Sports, University of Porto, Porto, Portugal. ${ }^{3}$ School of Physical Education and Sport of Ribeirão Preto, University of São Paulo, Ribeirão Preto, SP, Brazil. ${ }^{4}$ Physical Education Course, Paulista University, São José do Rio Preto, SP, Brazil. ${ }^{5}$ School of Medicine, Autonomous University of Madrid, Madrid, Spain. ${ }^{6}$ Faculty of Phycology, Education and Sport, University Lusófona of Porto, Porto, Portugal.

Received: 5 August 2021 Accepted: 17 November 2021

Published online: 18 December 2021

\section{References}

1. Bohannon RW. Hand-grip dynamometry predicts future outcomes in aging adults. J Geriatr Phys Ther. 2001;2008(31):3-10. https://doi.org/10. 1519/00139143-200831010-00002.

2. Teng Z, Zhu Y, Yu X, Liu J, Long Q, Zeng Y, et al. An analysis and systematic review of sarcopenia increasing osteopenia risk. PLOS ONE. 2021;16: e0250437. https://doi.org/10.1371/journal.pone.0250437.

3. Bohannon RW. Grip strength: an indispensable biomarker for older adults. Clin Interv Aging. 2019;14:1681-91. https://doi.org/10.2147/cia.s194543.

4. Santanasto AJ, Miljkovic I, Cvejkus RK, Wheeler VW, Zmuda JM. Sarcopenia characteristics are associated with incident mobility limitations in african caribbean men: the tobago longitudinal study of aging. J Gerontol A Biol Sci Med Sci. 2020;75:1346-52. https://doi.org/10.1093/gerona/glz233.

5. Landi F, Liperoti R, Russo A, Capoluongo E, Barillaro C, Pahor M, et al. Disability, more than multimorbidity, was predictive of mortality among older persons aged 80 years and older. J Clin Epidemiol. 2010;63:752-9. https://doi.org/10.1016/j.jclinepi.2009.09.007.

6. Clark BC, Manini TM. Sarcopenia $\neq$ Dynapenia. J Gerontol Ser A. 2008;63:829-34. https://doi.org/10.1093/gerona/63.8.829.

7. Fried LP, Tangen CM, Walston J, Newman AB, Hirsch C, Gottdiener J, et al. Frailty in older adults evidence for a phenotype. J Gerontol A Biol Sci Med Sci. 2001;56:M146-57.

8. Cruz-Jentoft AJ, Bahat G, Bauer J, Boirie Y, Bruyère O, Cederholm T, et al. Sarcopenia: revised European consensus on definition and diagnosis. Age Ageing. 2018;48:16-31. https://doi.org/10.1093/ageing/afy169.

9. Dodds RM, Syddall HE, Cooper R, Benzeval M, Deary IJ, Dennison EM, et al Grip strength across the life course: normative data from twelve British studies. PLoS ONE. 2014;9: e113637. https://doi.org/10.1371/journal.pone. 0113637.

10. Lauretani F, Russo CR, Bandinelli S, Bartali B, Cavazzini C, Di lorio A, et al. Age-associated changes in skeletal muscles and their effect on mobility: an operational diagnosis of sarcopenia. J Appl Physiol. 2003;95:1851-60.

11. Wang YC, Bohannon RW, Li X, Sindhu B, Kapellusch J. Hand-Grip Strength: Normative Reference Values and Equations for Individuals 18 to 85 Years of Age Residing in the United States. J Orthop Sports Phys Ther. 2018:48:685-93. https://doi.org/10.2519/jospt.2018.7851.

12. Albrecht BM, Stalling I, Bammann K. Sex- and age-specific normative values for handgrip strength and components of the Senior Fitness Test in community-dwelling older adults aged 65-75 years in Germany: results from the OUTDOOR ACTIVE study. BMC Geriatr. 2021;21:273. https://doi. org/10.1186/s12877-021-02188-9.

13. Hofmann M, Halper B, Oesen S, Franzke B, Stuparits P, Tschan H, et al. Serum concentrations of insulin-like growth factor-1, members of the TGF-beta superfamily and follistatin do not reflect different stages of dynapenia and sarcopenia in elderly women. Exp Gerontol. 2015;64:3545. https://doi.org/10.1016/j.exger.2015.02.008.

14. Lima RM, de Oliveira RJ, Raposo R, Neri SGR, Gadelha AB. Stages of sarcopenia, bone mineral density, and the prevalence of osteoporosis in older women. Arch Osteoporos. 2019;14:38. https://doi.org/10.1007/ s11657-019-0591-4

15. Akpinar TS, Tayfur M, Tufan F, Sahinkaya T, Kose M, Ozsenel EB, et al. Uncomplicated diabetes does not accelerate age-related sarcopenia. Aging Male Off J Int Soc Study Aging Male. 2014;17:205-10. https://doi. org/10.3109/13685538.2014.963040

16. Farinatti P, Paes L, Harris EA, Lopes GO, Borges JP. A simple model to identify risk of sarcopenia and physical disability in HIV-infected patients. 
J Strength Cond Res. 2017;31:2542-51. https://doi.org/10.1519/jsc.00000 00000002070

17. Gadelha AB, Vainshelboim B, Ferreira AP, Neri SGR, Bottaro M, Lima RM. Stages of sarcopenia and the incidence of falls in older women: a prospective study. Arch Gerontol Geriatr. 2018;79:151-7. https://doi.org/10. 1016/j.archger.2018.07.014.

18. McGrath R, Hackney KJ, Ratamess NA, Vincent BM, Clark BC, Kraemer WJ. Absolute and body mass index normalized handgrip strength percentiles by gender, ethnicity, and hand dominance in Americans. Adv Geriatr Med Res. 2020. https://doi.org/10.20900/agmr20200005.

19. Manini TM, Visser M, Won-Park S, Patel KV, Strotmeyer ES, Chen H, et al. Knee extension strength cutpoints for maintaining mobility. J Am Geriatr Soc. 2007:55:451-7. https://doi.org/10.1111/j.1532-5415.2007.01087.x.

20. de Mello RGB, Dalla Corte RR, Gioscia J, Moriguchi EH. Effects of physical exercise programs on sarcopenia management, dynapenia, and physical performance in the elderly: a systematic review of randomized clinical trials. J Aging Res. 2019;2019:1959486. https://doi.org/10.1155/2019/19594 86.

21. Sardeli AV, Komatsu TR, Mori MA, Gáspari AF, Chacon-Mikahil MPT. Resistance training prevents muscle loss induced by caloric restriction in obese elderly individuals: a systematic review and meta-analysis. Nutrients. 2018:10:423.

22. Strasser EM, Hofmann M, Franzke B, Schober-Halper B, Oesen S, Jandrasits $W$, et al. Strength training increases skeletal muscle quality but not muscle mass in old institutionalized adults: a randomized, multi-arm parallel and controlled intervention study. Eur J Phys Rehabil Med. 2018;54:92133. https://doi.org/10.23736/s1973-9087.18.04930-4.

23. Foley KT, Owings TM, Pavol MJ, Grabiner MD. Maximum grip strength is not related to bone mineral density of the proximal femur in older adults. Calcif Tissue Int. 1999;64:291-4.

24. Maranhao Neto GA, Oliveira AJ, Pedreiro RC, Pereira-Junior PP, Machado $\mathrm{S}$, Marques Neto S, et al. Normalizing handgrip strength in older adults: an allometric approach. Arch Gerontol Geriatr. 2017;70:230-4. https://doi. org/10.1016/j.archger.2017.02.007.

25. Pua Y-H. Allometric analysis of physical performance measures in older adults. Phys Ther. 2006;86:1263-70.

26. Abdalla PP, Carvalho AS, Santos AP, Venturini ACR, Alves TC, Mota J, et al. Cut-off points of knee extension strength allometrically adjusted to identify sarcopenia risk in older adults: a cross-sectional study. Arch Gerontol Geriatr. 2020;89: 104100. https://doi.org/10.1016/j.archger.2020.104100.

27. Abdalla PP, Venturini ACR, Santos APD, Tasinafo M, Marini JAG, Alves TC, et al. Normalizing calf circumference to identify low skeletal muscle mass in older women: a cross-sectional study. Nutr Hosp. 2021;38:7. https://doi. org/10.20960/nh.03572.

28. Davies MJ, Dalsky GP. Normalizing strength for body size differences in older adults. Med Sci Sports Exerc. 1997;29:713-7.

29. Bouchard DR, Beliaeff S, Dionne IJ, Brochu M. Fat mass but not fat-free mass is related to physical capacity in well-functioning older individuals: nutrition as a determinant of successful aging (NuAge) — the Quebec Longitudinal Study. J Gerontol Ser A. 2007;62:1382-8. https://doi.org/10. 1093/gerona/62.12.1382.

30. Broadwin J, Goodman-Gruen D, Slymen D. Ability of fat and fat-free mass percentages to predict functional disability in older men and women. J Am Geriatr Soc. 2001;49:1641-5. https://doi.org/10.1111/j.1532-5415. 2001.49273.x

31. Enright PL. The six-minute walk test. Respir Care. 2003;48:783-5.

32. Cuschieri S. The STROBE guidelines. Saudi J Anaesth. 2019;13:S31-4. https://doi.org/10.4103/sja.SJA_543_18.

33. Bolfarine H, Bussab WO. Elementos de amostragem. São Paulo: Edgard Blücher; 2005.

34. Binder EF, Yarasheski KE, Steger-May K, Sinacore DR, Brown M, Schechtman KB, et al. Effects of progressive resistance training on body composition in frail older adults: results of a randomized, controlled trial. J Gerontol Ser A. 2005:60:1425-31.

35. Cruz-Jentoft AJ, Landi F, Schneider SM, Zuniga C, Arai H, Boirie Y, et al. Prevalence of and interventions for sarcopenia in ageing adults: a systematic review. Report of the International Sarcopenia Initiative (EWGSOP and IWGS). Age Ageing. 2014;43:748-59. https://doi.org/10.1093/ageing/ afu115.

36. Finney GR, Minagar A, Heilman KM. Assessment of mental status. Neurol Clin. 2016;34:1-16. https://doi.org/10.1016/j.ncl.2015.08.001.
37. Icaza MC, Albala C. Projeto SABE. Minimental State Examination (MMSE) del estudio de dementia en Chile: análisis estatístico Brasília: OPAS 1999:1-18.

38. WHO Expert Consultation. Appropriate body-mass index for Asian populations and its implications for policy and intervention strategies. Lancet (Lond, Engl). 2004;363:157-63. https://doi.org/10.1016/s0140-6736(03) 15268-3.

39. Segal NA, Torner JC, Yang M, Curtis JR, Felson DT, Nevitt MC. Muscle mass is more strongly related to hip bone mineral density than is quadriceps strength or lower activity level in adults over age 50 year. J Clin Densitom. 2008;11:503-10. https://doi.org/10.1016/j.jocd.2008.03.001.

40. Bailey BJ, Briars GL. Estimating the surface area of the human body. Stat Med. 1996;15:1325-32.

41. Jelliffe DB, Jelliffe EP. The arm circumference as a public health index of protein-calorie malnutrition of early childhood. 20. Current conclusions. J Trop Pediatr. 1969;15:253-60.

42. Heymsfield SB, McManus C, Smith J, Stevens V, Nixon DW. Anthropometric measurement of muscle mass: revised equations for calculating bone-free arm muscle area. Am J Clin Nutr. 1982;36:680-90.

43. George LB, Bruce RB, Baltej SM, Haran TS, Michael FS. Nutritional and metabolic assessment of the hospitalized patient. J Parenter Enter Nutr. 1977;1:11-21. https://doi.org/10.1177/014860717700100101.

44. Lean M, Han TS, Deurenberg P. Predicting body composition by densitometry from simple anthropometric measurements. Am J Clin Nutr. 1996;63:4-14.

45. Baumgartner RN, Koehler KM, Gallagher D, Romero L, Heymsfield SB, Ross RR, et al. Epidemiology of sarcopenia among the elderly in New Mexico. Am J Epidemiol. 1998;147:755-63.

46. Baumgartner RN, Heymsfield SB, Lichtman S, Wang J, Pierson RN Jr. Body composition in elderly people: effect of criterion estimates on predictive equations. Am J Clin Nutr. 1991;53:1345-53. https://doi.org/10.1093/ajcn/ 53.6.1345.

47. Lohman TG, Roche AF, Martorell R. Anthropometric standardization reference manual. Hanover: Human Kinetics Books; 1988.

48. Morley JE, Abbatecola AM, Argiles JM, Baracos V, Bauer J, Bhasin S, et al. Sarcopenia with limited mobility: an international consensus. J Am Med Dir Assoc. 2011;12:403-9. https://doi.org/10.1016/j.jamda.2011.04.014.

49. Massy-Westropp NM, Gill TK, Taylor AW, Bohannon RW, Hill CL. Hand Grip Strength: age and gender stratified normative data in a population-based study. BMC Res Notes. 2011;4:1-5.

50. Lourenco R, Perez-Zepeda M, Gutierrez-Robledo L, Rodriguez Manas L, Garcia-Garcia F. Performance of the European Working Group on Sarcopenia in Older People algorithm in screening older adults for muscle mass assessment. Age Ageing. 2014;44:334-8. https://doi.org/10.1093/ ageing/afu192.

51. Alexandre S, Duarte YA, Santos JL, Wong R, Lebrao ML. Prevalence and associated factors of sarcopenia among elderly in Brazil: findings from the SABE study. J Nutr Health Aging. 2014;18:284-90. https://doi.org/10.1007/ s12603-013-0413-0.

52. Brzycki M. Strength testing - predicting a one-rep max from reps-tofatigue. J Phys Educ Recreat Dance. 1993;64:88-90.

53. Abdalla PP, Carvalho AS, Santos AP, Venturini ACR, Alves TC, Mota J, et al. One-repetition submaximal protocol to measure knee extensor muscle strength among older adults with and without sarcopenia: a validation study. BMC Sports Sci Med Rehabil. 2020;12:29. https://doi.org/10.1186/ s13102-020-00178-9.

54. Matsudo S, Araújo T, Matsudo V, Andrade D, Andrade E, Oliveira LC, et al. Questionário internacional De atividade física (ipaq): estupo De validade e reprodutibilidade No Brasil. Revista Brasileira de Atividade Física \& Saúde. 2012;6:5-18.

55. Myers R. Classical and modern regression with applications. Boston: PWS and Kent Publishing Company. Inc; 1990.

56. Jaric $\mathrm{S}$. Role of body size in the relation between muscle strength and movement performance. Exerc Sport Sci Rev. 2003;31:8-12.

57. Mukaka MM. Statistics corner: A guide to appropriate use of correlation coefficient in medical research. Malawi Med J. 2012;24:69-71.

58. Kwak SG, Kim JH. Central limit theorem: the cornerstone of modern statistics. Korean J Anesthesiol. 2017;70:144-56. https://doi.org/10.4097/kjae. 2017.70.2.144. 
59. Schisterman EF, Perkins NJ, Liu A, Bondell H. Optimal cut-point and its corresponding Youden Index to discriminate individuals using pooled blood samples. Epidemiology. 2005;16:73-81.

60. Hosmer D, Lemeshow S. Applied logistic regression. 2nd ed. Nova Jersey, EUA:Wiley; 2000.

61. Cawthon PM, Manini T, Patel SM, Newman A, Travison T, Kiel DP, et al. Putative cut-points in sarcopenia components and incident adverse health outcomes: an SDOC analysis. J Am Geriatr Soc. 2020;68:1429-37. https://doi.org/10.1111/jgs.16517.

62. Alley DE, Shardell MD, Peters KW, McLean RR, Dam T-TL, Kenny AM, et al. Grip strength cutpoints for the identification of clinically relevant weakness. J Gerontol Ser A. 2014;69:559-66. https://doi.org/10.1093/gerona/ glu011.

63. Owings TM, Pavol MJ, Grabiner MD. Lower extremity muscle strength does not independently predict proximal femur bone mineral density in healthy older adults. Bone. 2002;30:515-20.

64. Ramírez-Vélez R, de Asteasu MLS, Martínez-Velilla N, Zambom-Ferraresi F, García-Hermoso A, Izquierdo M. Handgrip strength as a complementary test for mobility limitations assessment in acutely hospitalized oldest old. Rejuvenation Res. 2021. https://doi.org/10.1089/rej.2020.2344.

\section{Publisher's Note}

Springer Nature remains neutral with regard to jurisdictional claims in published maps and institutional affiliations.

- fast, convenient online submission

- thorough peer review by experienced researchers in your field

- rapid publication on acceptance

- support for research data, including large and complex data types

- gold Open Access which fosters wider collaboration and increased citations

- maximum visibility for your research: over $100 \mathrm{M}$ website views per year

At BMC, research is always in progress.

Learn more biomedcentral.com/submissions 